\title{
GENERALIZED DEGREE THEORY FOR SEMILINEAR OPERATOR EQUATIONS
}

\author{
YUQING CHEN \\ Department of Mathematics, Foshan University, Foshan, Guangdong 528000, P. R. China \\ e-mail:yqchen@foshan.net \\ and DONAL O'REGAN \\ Department of Mathematics, National University of Ireland, Galway, Ireland \\ e-mail:donal.oregan@nuigalway.ie
}

(Received 17 May, 2005; accepted 14 October, 2005)

\begin{abstract}
In this paper, we construct a generalized degree theory of BrowderPetryshyn or Petryshyn type for a class of semilinear operator equations involving a Fredholm type mapping with infinite dimensional kernel.

2000 Mathematics Subject Classification. Primary 47H05, 47H11; Secondary 35L05, 55M25.
\end{abstract}

1. Introduction and Preliminaries. In this paper, we study the following semilinear operator equation

$$
L x-N x=f, x \in X, f \in Y,
$$

where $X, Y$ are Banach spaces, and $L: D(L) \subset X \rightarrow Y$ is a linear Fredholm type mapping with $\operatorname{dim}(\operatorname{Ker}(\mathrm{L}))=+\infty$, and $N: \bar{\Omega} \cap D(L) \rightarrow Y$ is a nonlinear mapping. This type of map equation has been extensively studied by Mawhin, Petryshyn and others for the case when $\operatorname{dim}(\operatorname{Ker}(\mathrm{L}))<+\infty$, see [8], [12] for references. By imposing some suitable conditions on $X, L$ and $Y$, we can apply Browder-Petryshyn's degree and Petryshyn's generalized degree theory to study such an equation. A generalized degree theory for $L-N$ is defined in three ways by following Browder-Petryshyn and Petryshyn's method or combining them with Mawhin's method. First we recall some definitions.

DefinITION 1.1. [12] Let $X$ be a real separable Banach space, $\left(X_{n}\right)_{n=1}^{\infty}$ a sequence of finite dimensional subspaces of $X$, and $P_{n}: X \rightarrow X_{n}$ a projecton for $n=1,2, \ldots$ If $P_{n} x \rightarrow x$ as $n \rightarrow \infty$, for all $x \in X$, then $\left\{X_{n}, P_{n}\right\}$ is called a projectionally complete scheme for $X$.

Definition 1.2. [12] Let $X, Y$ be two real separable Banach spaces, $\left(X_{n} \subset\right.$ $X)_{n=1}^{\infty},\left(Y_{n} \subset Y\right)_{n=1}^{\infty}$ two sequences of oriented finite dimensional subspaces such that $\operatorname{dim}\left(X_{n}\right)=\operatorname{dim}\left(Y_{n}\right)$, and let $Q_{n}: Y \rightarrow Y_{n}$ be a linear mapping of $Y$ onto $Y_{n}$ for $n=1,2 \ldots$ If $\lim _{n \rightarrow \infty} d\left(x, X_{n}\right)=0$, and $\left(Q_{n}\right)$ is uniformly bounded, then we call $\Gamma_{A}=\left\{X_{n}, Y_{n}, Q_{n}\right\}$ an admissible scheme for $(X, Y)$; if $Q_{n}$ is the projection such that $Q_{n} y \rightarrow y$ for all $y \in Y$, then we say $\Gamma_{0}=\left\{X_{n}, Y_{n}, Q_{n}\right\}$ is a projectionally complete scheme for $(X, Y)$. 
DEFINITION 1.3. [12] Let $X, Y$ be real separable Banach spaces with a projectionally complete scheme $\Gamma_{0}=\left\{X_{n}, Y_{n}, Q_{n}\right\}, D \subset X$, and $T: D \rightarrow Y$. Suppose that the following conditions are satisfied:

(1) $Q_{n} T: D \cap X_{n} \rightarrow Y_{n}$ is continuous for $n=1,2 \ldots$;

(2) for any bounded sequence $\left(x_{n_{j}} \in X_{n_{j}} \cap D\right)_{j=1}^{\infty}$ such that $Q_{n_{j}} T x_{n_{j}} \rightarrow y$, there exists a subsequence $\left(x_{n_{j}}^{\prime}\right)$ such that $x_{n_{j}}^{\prime} \rightarrow x \in D$ and $T x=y$;

then $T$ is said to be A-proper with respect to $\Gamma_{0}$; if (2) is replaced by the following

(3) for any bounded sequence $\left(x_{n_{j}} \in X_{n_{j}} \cap D\right)_{j=1}^{\infty}$ such that $Q_{n_{j}} T x_{n_{j}} \rightarrow y$, there exists $x \in D$ such that $T x=y$

then $T$ is said to be pseudo A-proper with respect to $\Gamma_{0}$.

Definition 1.4. Let $X, Y$ be two real Banach spaces, $L: D(L) \subseteq X \rightarrow Y$ is a linear mapping, and we say $L$ is a Fredholm mapping of index zero type if

(1) $\operatorname{Ker}(L)=\{x \in X: L x=0\}, \operatorname{Im}(L)=\{L x: x \in D(L)\}$ are closed in $H$;

(2) $X=\operatorname{Ker}(L) \oplus X_{1}$ for some subspace $X_{1}$ of $X, Y=Y_{1} \oplus \operatorname{Im}(L)$ for some subspace $Y_{1}$ of $Y$;

(3) $\operatorname{Ker}(L)$ is linearly homeomorphic to $\operatorname{Coker}(L)=Y / \operatorname{Im}(L)$.

REMARK 1. Obviously, if $X$ is linearly homeomorphic to $Y, L=0$ is a Fredholm mapping of index zero type, but not a Fredholm mapping of index zero. If $L$ is a Fredholm mapping of index zero, then $\operatorname{dim}(\operatorname{Ker}(L))=\operatorname{dim}(\operatorname{Coker}(L))<+\infty$, and so $\operatorname{Ker}(L)$ is linearly homeomorphic to $\operatorname{Coker}(L)$; thus $L$ is a Fredholm mapping of index zero type.

Now, assume that $L: D(L) \subset X \rightarrow Y$ is a Fredholm mapping of index zero type. Then there exist linear projections $P: X \rightarrow X$ and $Q: Y \rightarrow Y$ such that $\operatorname{Im}(P)=$ $\operatorname{Ker}(L)$ and $\operatorname{Im}(Q)=Y_{1}$.

Obviously, the restriction of $L_{P}$ of $L$ to $D(L) \cap \operatorname{Ker}(P)$ is one to one and onto $\operatorname{Im}(L)$, so its inverse $K_{P}: \operatorname{Im}(L) \rightarrow D(L) \cap \operatorname{Ker}(P)$ is defined. Let $J: \operatorname{Ker}(L) \rightarrow Y_{1}$ be a linear homeomorphism, and set $K_{P Q}=K_{P}(I-Q)$.

Proposition 1.5. $L+\lambda J P: X \rightarrow Y$ is a bijective mapping for each $\lambda \neq 0$.

Proof. For each $\lambda \neq 0$, if $L x+\lambda J P x=0$, then $J P x=0, L x=0$, so $x \in \operatorname{Ker}(L)$, thus $x=0$. On the other hand, for $y=y_{1}+y_{2} \in Y, y_{1} \in Y_{1}, y_{2} \in \operatorname{Im}(L)$, put $x=$ $\lambda^{-1} J^{-1} y_{1}+K_{P} y_{2}$, then $L x+\lambda J P x=y$. Therefore $L+\lambda J P$ is bijective.

Proposition 1.6. Let $X, Y$ be real separable Banach spaces, and $\left(Y_{n}, Q_{n}\right) a$ projectionally complete scheme for $Y$, and let $L: D(L) \subset X \rightarrow Y$ be a Fredholm mapping of zero index type. Then for each $\lambda \neq 0$, there exists a projectionally complete scheme $\Gamma_{\lambda, L}$ for $(X, Y)$.

Proof. For each $\lambda \neq 0$, put $K_{\lambda}=L+\lambda J P$. By Proposition $1.5, K_{\lambda}$ is bijiective. Set $X_{n}=K_{\lambda}^{-1} Y_{n}$ for $n=1,2 \ldots$ Obviously, we have $\operatorname{dim}\left(X_{n}\right)=\operatorname{dim}\left(Y_{n}\right)$, and $X=\overline{\cup_{n=1}^{\infty} X_{n}}$. Thus $\Gamma_{L}=\left\{X_{n}, Y_{n}, Q_{n}\right\}$ is a projectionally complete scheme for $(X, Y)$.

Petryshyn showed that if $L$ is a Fredhom mapping of index zero, then $L$ is A-proper with respect to $\Gamma_{1, L}$, see [12]. Here we have a similar result.

Proposition 1.7. Let $L: D(L) \subset X \rightarrow Y$ be a Fredholm mapping of zero index type, and assume that $X$ is reflexive. If $G \subset X$ is bounded closed convex, then $L: G \cap D(L) \rightarrow$ $Y$ is pseudo A-proper with respect to $\Gamma_{\lambda, L}$ for each $\lambda \neq 0$. 
Proof. For any sequence $x_{n_{k}} \in G \cap D(L) \cap X_{n_{k}}$ with $Q_{n_{k}} L x_{n_{k}} \rightarrow y$, we may assume that $x_{n_{k}} \rightarrow x_{0} \in G$ by taking a subsequence.

Notice that $Q_{n_{k}}\left(L x_{n_{k}}+\lambda J P x_{n_{k}}\right)=L x_{n_{k}}+\lambda J P x_{n_{k}}$, and $J P x_{n_{k}} \rightarrow J P x_{0}$, so we have

$$
x_{n_{k}}=(L+J P)^{-1}\left(Q_{n_{k}}\left(L x_{n_{k}}+J P x_{n_{k}}\right)\right) \rightarrow(L+J P)^{-1}\left(y+J P x_{0}\right)=x_{0} .
$$

Thus $x_{0} \in D(L)$, and $L x_{0}=y$, so therefore $L$ is pseudo A-proper with respect to $\Gamma_{\lambda, L}$.

Definition 1.8. Let $X$ be a real separable Banach space and $\Gamma_{0}=\left(X_{n}, P_{n}\right)$ a projectionally complete scheme for $X, Y$ a real Banach space, $L: D(L) \subset X \rightarrow Y$ a Fredholm mapping of zero index type, and let $N: D \subset X \rightarrow Y$ be a mapping.

(1) If $I-P-\left(J^{-1} Q+K_{P Q}\right) N$ is A-proper with respect to $\Gamma_{0}$, then we say $N$ is $L$-A-proper with respect to $\Gamma_{0}$;

(2) If $I-P-\left(J^{-1} Q+K_{P Q}\right) N$ is pseudo A-proper with respect to $\Gamma_{0}$, then we say $N$ is pseudo $L$-A-proper with respect to $\Gamma_{0}$;

(3) A family of mappings $H(t, x):[0,1] \times D \rightarrow Y$ is called a homotopy of $L-A$ proper mappings with respect to $\Gamma_{0}$ if $H(t, \cdot)$ is an $L$-A-proper mapping with respect to $\Gamma_{0}$ for each $t \in[0,1]$.

Proposition 1.9. Let $L: D(L) \subseteq X \rightarrow Y$ be a linear mapping with $\operatorname{Ker}(L)=\{0\}$, and $\operatorname{Im}(L)=Y$. Then the following conclusions hold

(1) if $\Gamma_{0}=\left(X_{n}, P_{n}\right)$ is a projectionally complete scheme for $X$, then 0 is $L$-A-proper with respect to $\Gamma_{0}$;

(2) if $\left(Y_{n}, Q_{n}\right)$ is a projectionally complete scheme for $Y$, and $L^{-1}$ is continuous, then $L$ is A-proper with respect to $\Gamma_{1, L}$, where $\Gamma_{1, L}$ is constructed as in Proposition 1.6.

Proof. (1) We have $P=0$, and $Q=0$, and the identity mapping $I: X \rightarrow X$ is obviously A-proper with respect to $\Gamma_{0}$. Thus 0 is $L$-A-proper with respect to $\Gamma_{0}$.

(2) Since $\operatorname{Ker}(L)=\{0\}$, the mapping $K$ in the proof of Proposition 1.6 is just the mapping $L$, so $X_{n}=L^{-1} Y_{n}$. If $x_{n_{k}} \in X_{n_{k}}$ such that $Q_{n_{k}} L x_{n_{k}} \rightarrow y$, then $L x_{n_{k}}=$ $Q_{n_{k}} L x_{n_{k}} \rightarrow y$. Therefore we have $x_{n_{k}} \rightarrow L^{-1} y$. The conclusion holds.

Proposition 1.10. Let $L: D(L) \subset X \rightarrow Y$ be a Fredholm mapping of zero index type, $\Gamma_{0}=\left(X_{n}, P_{n}\right)$ a projectionally complete scheme for $X, G \subset X$ a bounded closed convex subset, and $T: G \rightarrow Y$ a weakly continuous mapping, with $X$ reflexive. Then $T$ is L-pseudo A-proper with respect to $\Gamma_{0}$.

Proof. For any subsequence $x_{n_{k}} \in X_{n_{k}}$ such that $P_{n_{k}}\left(I-P-J^{-1} Q T-\right.$ $\left.K_{P Q} T\right) x_{n_{k}} \rightarrow y$, we may assume that $x_{n_{k}} \rightarrow x_{0} \in G$ by taking a subsequence, and so $(I-P) x_{n_{k}} \rightarrow x_{0}, J^{-1} Q T x_{n_{k}} \rightarrow J^{-1} Q T x_{0}$, and $K_{P Q} T x_{n_{k}} \rightarrow K_{P Q} T x_{0}$. Consequently, $\left(I-P-J^{-1} Q T-K_{P Q} T\right) x_{0}=y$, so $T$ is $L$-pseudo A-proper with respect to $\Gamma_{0}$.

Proposition 1.11. Let $X, Y$ be real separable Banach spaces, and $\left(Y_{n}, Q_{n}\right)$ a projectionally complete scheme for $Y$. Let $L: D(L) \subset X \rightarrow Y$ be a Fredholm mapping of zero index type, $G \subset X$ a bounded closed subset, and $N: G \rightarrow Y$ a continuous compact mapping. Then $L+\lambda J P-N$ is A-proper with respect to $\Gamma_{\lambda, L}$ for each $\lambda>0$.

Proof. For any sequence $x_{n_{k}} \in G \cap D(L) \cap X_{n_{k}}$ with $Q_{n_{k}}(L+\lambda J P-N) x_{n_{k}} \rightarrow y$, in view of the compactness of $N$, we may assume that $N x_{n_{k}} \rightarrow y_{0} \in Y$ by taking a subsequence. 
Notice that $Q_{n_{k}}\left(L x_{n_{k}}+\lambda J P x_{n_{k}}\right)=L x_{n_{k}}+\lambda J P x_{n_{k}}$, so we have

$$
x_{n_{k}}=(L+J P)^{-1}\left[Q_{n_{k}}(L+\lambda J P-N) x_{n_{k}}+Q_{n_{k}} N x_{n_{k}}\right] \rightarrow(L+\lambda J P)^{-1}\left(y+y_{0}\right)=x_{0} .
$$

Thus $x_{0} \in D(L)$, and $N x_{0}=y_{0},(L+\lambda J P-N) x_{0}=y$, and therefore $L$ is A-proper with respect to $\Gamma_{\lambda, L}$.

2. Generalized degree theory for $L-N$. In this section, $X, Y$ are real separable Banach spaces, $L: D(L) \subseteq X \rightarrow Y$ is a Fredholm mapping of index zero type with $D(L)$ dense in $X$, and $N: \bar{\Omega} \subset X \rightarrow Y$ is a nonlinear mapping, and we consider the semilinear operator equation $L x-N x=0$. We will apply Browder-Petryshyn and Petryshyn's generalized degree theory to study such an equation in three different ways.

LeMma 2.1. Let $L: D(L) \subseteq X \rightarrow Y$ be a Fredholm mapping of index zero type, and $\Omega \subset X$ an open bounded subset, and let $N: \bar{\Omega} \rightarrow Y$ be a mapping. If $0 \notin(L-N)(\partial \Omega \cap$ $D(L))$, then $0 \notin\left[I-P-\left(J^{-1} Q+K_{P Q}\right) N\right](\partial \Omega)$.

Proof. Suppose the contrary i.e. suppose there exists $x_{0} \in \partial \Omega$ such that $0 \in x_{0}-$ $P x_{0}-\left(J^{-1} Q+K_{P Q}\right) N x_{0}$. Since $J^{-1} Q T x_{0} \in \operatorname{Ker}(L)=\operatorname{Im}(P), x_{0}-P x_{0} \in \operatorname{Ker}(P)$, and $K_{p Q} T x_{0} \in D(L) \cap \operatorname{Ker}(P)$, we must have

$$
J^{-1} Q N x_{0}=0, \quad x_{0}-P x_{0}-K_{P Q} N x_{0}=0 .
$$

Therefore we have

$$
Q N x_{0}=0, \quad x_{0}-P x_{0}-K_{P} N x_{0}=0 \text {, i.e. } L x_{0}-N x_{0}=0,
$$

which is a contradiction to $0 \notin(L-N)(\partial \Omega \cap D(L))$.

Now, let $L: D(L) \subseteq X \rightarrow Y$ be a Fredholm mapping of index zero type, $\Gamma_{0}=$ $\left(X_{n}, P_{n}\right)$ a projectionally complete scheme for $X$ and $\Omega \subset X$ an open bounded subset, and let $N: \bar{\Omega} \rightarrow Y$ be an $L$-A-proper mapping with respect to $\Gamma_{0}$. Suppose $0 \notin(L-T)(\partial \Omega \cap D(L))$. By Lemma 2.1, $0 \notin\left[I-P-\left(J^{-1} Q+K_{P Q}\right) N\right](\partial \Omega)$. Since $I-P-\left(J^{-1} Q+K_{P Q}\right) N$ is an A-proper mapping with respect to $\Gamma_{0}$, the generalized degree $\operatorname{deg}\left(I-P-\left(J^{-1} Q+K_{P Q}\right) N, \Omega, 0\right)$ is well defined, see [3], and we define

$$
\operatorname{deg}_{\Gamma_{0}, J}(L-N, \Omega, 0)=\operatorname{deg}\left(I-P-\left(J^{-1} Q+K_{P Q}\right) N, \Omega, 0\right),
$$

which is called the generalized coincidence degree of $L$ and $N$ on $\Omega$.

THEOREM 2.2. The generalized coincidence degree of $L$ and $N$ defined by (2.1) on $\Omega$ has the following properties.

(1) If $\Omega_{1}$ and $\Omega_{2}$ are disjoint open subsets of $\Omega$ such that 0 does not belong to $(L-N)\left(D(L) \cap \overline{\left.\Omega \backslash\left(\Omega_{1} \cup \Omega_{2}\right)\right)}\right.$, then

$$
\operatorname{deg}_{\Gamma_{0}, J}(L-N, \Omega, 0) \subseteq \operatorname{deg}_{\Gamma_{0}, J}\left(L-N, \Omega_{1}\right)+\operatorname{deg}_{\Gamma_{0}, J}\left(L-N, \Omega_{2}, 0\right) .
$$

(2) If $H(t, x):[0,1] \times \bar{\Omega} \rightarrow Y$ is a homotopy of L-A-proper mappings with respect to $\Gamma_{0}$, and if $0 \neq L x-H(t, x)$ for all $(t, x) \in[0,1] \times \partial \Omega \cap D(L)$, then $\operatorname{deg}_{\Gamma_{0}, J}(L-H(t, \cdot), \Omega, 0)$ does not depend on $t \in[0,1]$. 
(3) If $\operatorname{deg}_{\Gamma_{0}}(L-N, \Omega, 0) \neq\{0\}$, then $0 \in(L-N)(D(L) \cap \Omega)$.

(4) If $L: D(L) \subseteq X \rightarrow Y$ is a linear mapping such that $L^{-1}: Y \rightarrow D(L)$ is continuous, then $\operatorname{deg}_{\Gamma_{0}, J}(L, \Omega, 0)=\{1\}$ if $0 \in \Omega$.

(5) If $\Omega$ is a symmetric neighbourhood of 0 , and $N: \bar{\Omega} \rightarrow Y$ is an odd L-A-proper mapping with respect to $\Gamma_{0}$ with $0 \notin(L-N)(\partial \Omega \cap D(L))$, then $\operatorname{deg}_{\Gamma_{0}, J}(L-N, \Omega, 0)$ does not contain even numbers.

Proof. (1)-(3) follow directly from the definition and the properties of generalized degree.

(4) Since $\operatorname{Ker}(L)=\{0\}, P=0, Q=0$, the zero mapping is $L$-A-proper with respect to $\Gamma_{0}$. Thus $\operatorname{deg}_{\Gamma_{0}, J}(L, \Omega, 0)=\operatorname{deg}(I, \Omega, 0)=\{1\}$.

(5) Since $N$ is odd, the mapping $I-P-\left(J^{-1} Q+K_{P Q}\right) N$ is odd. Thus $\operatorname{deg}(I-$ $\left.P-\left(J^{-1} Q+K_{P Q}\right) N, \Omega, 0\right)$ does not contain even numbers, and the conclusion follows by definition.

COROLlary 2.3. Let $L: D(L) \subseteq X \rightarrow Y$ be a linear mapping such that $L^{-1}: Y \rightarrow$ $D(L)$ is continuous, $\Omega \subset X$ an open bounded subset with $0 \in \Omega$, and $N: \bar{\Omega} \rightarrow Y$ a mapping such that $\{L-t N\}_{t \in[0,1]}$ is a homotopy of $L$-A-proper mappings with respect to $\Gamma_{0}$. If $L x \notin t N x$ for all $(t, x) \in[0,1] \times \partial \Omega \cap D(L)$, then $\operatorname{deg}(L-N, \Omega, 0)=1$.

In the following, let $L: D(L) \subset X \rightarrow Y$ be a densely defined Fredholm mapping of zero index type. We assume that $\Gamma_{0}=\left(Y_{n}, Q_{n}\right)$ is a projectionally complete scheme for $Y, \Gamma_{\lambda, L}$ is as defined in Proposition 1.6, and $L+\lambda J P-N$ is an A-proper map with respect to $\Gamma_{\lambda, L}$ for $\lambda \in\left(0, \lambda_{0}\right)$, where $\lambda_{0}>0$ is a constant. Suppose that $0 \notin$ $\overline{(L-N)(D(L) \cap \partial \Omega)}$. Then there exists $\lambda_{1}<\lambda_{0}$ such that $0 \notin(L+\lambda J P-N)(D(L) \cap$ $\partial \Omega)$ for all $\lambda \in\left(0, \lambda_{1}\right)$. We define a generalized degree

$$
\operatorname{deg}(L-N, \Omega, 0)=\cap_{0<\lambda<\lambda_{1}} \cup_{0<\epsilon \leq \lambda} \operatorname{deg}(L+\epsilon J P-N, \Omega, 0),
$$

where $\operatorname{deg}(L+\epsilon J P-N, \Omega, 0)$ is the generalized degree for A-proper maps with respect to $\Gamma_{\lambda, L}$, see [12].

Notice that if $0 \notin(L+\lambda J P-N)(D(L) \cap \partial \Omega)$ for all $\lambda \in\left(0, \lambda_{2}\right)$, then it is easy to check that

$$
\cap_{0<\lambda<\lambda_{1}} \cup_{0<\epsilon \leq \lambda} \operatorname{deg}(L+\epsilon J P-N, \Omega, 0)=\cap_{0<\lambda<\lambda_{2}} \cup_{0<\epsilon \leq \lambda} \operatorname{deg}(L+\epsilon J P-N, \Omega, 0) .
$$

Thus (2.2) is well defined.

REMARK. A degree theory for uniform limits of A-proper maps has been defined by P. M. Fitzpatrick [5]. Since $\Gamma_{\lambda, L}$ depends on $\lambda$, and $L+\lambda J P-N$ is an A-proper map with respect to $\Gamma_{\lambda, L}, L-N$ is slightly different to the uniform limits of A-proper maps. Of course, a slight generalization of the ideas in [5] could be applied here also.

THEOREM 2.4. The generalized degree defined by (2.2) has the following properties.

(1) If $\Omega_{1}$ and $\Omega_{2}$ are two open subsets of $\Omega$ such that $\Omega_{1} \cap \Omega_{2}=\emptyset$, and $0 \notin$ $\overline{(L-N)\left(D(L) \cap \overline{\Omega \backslash\left(\Omega_{1} \cup \Omega_{2}\right)}\right)}$, then

$$
\operatorname{deg}(L-N, \Omega, 0) \subseteq \operatorname{deg}\left(L-N, \Omega_{1}\right)+\operatorname{deg}\left(L-N, \Omega_{2}, 0\right) .
$$

(2) If $H(t, x):[0,1] \times \bar{\Omega} \rightarrow Y$ satisfies $0 \notin \overline{\cup_{t \in[0,1]}(L-H(t, \cdot))(D(L) \cap \partial \Omega)}$, and $\{L+\lambda J P-H(t, \cdot)\}_{t \in[0,1]}$ is a homotopy of A-proper maps with respect to $\Gamma_{\lambda, L}$ for each 
$\lambda \in\left(0, \lambda_{0}\right)$, where $\lambda_{0}>0$ is a constant, then $\operatorname{deg}(L-H(t, \cdot), \Omega, 0)$ does not depend on $t \in[0,1]$.

(3) If $\operatorname{deg}_{\Gamma_{0}}(L-N, \Omega, 0) \neq\{0\}$, then $0 \in \overline{(L-N)(D(L) \cap \Omega)}$.

(4) If $\Omega$ is a symmetric neighbourhood of 0 , and $N: \bar{\Omega} \rightarrow Y$ is an odd mapping such that $L+\lambda J P-N$ is A-proper with respect to $\Gamma_{\lambda, L}$ for each $\lambda \in\left(0, \lambda_{0}\right)$, where $\lambda_{0}>0$ is a constant, and $0 \notin \overline{(L-N)(\partial \Omega \cap D(L))}$, then $\operatorname{deg}(L-N, \Omega, 0)$ does not contain even numbers.

(5) $\operatorname{deg}(L, \Omega, 0) \subseteq\{ \pm 1\}$ if $0 \in \Omega$.

Proof. (1). By assumption, there exists $\lambda_{0}>0$ such that

$$
0 \notin(L+\lambda J P-N)\left(D(L) \cap \overline{\Omega \backslash\left(\Omega_{1} \cup \Omega_{2}\right)}\right)
$$

for all $\lambda \in\left(0, \lambda_{0}\right)$. If $m \in \operatorname{deg}(L-N, \Omega, 0)$, then there exist $\lambda_{j} \rightarrow 0^{+}, \lambda_{j}<\lambda_{0}, j=$ $1,2, \ldots$, such that $m \in \operatorname{deg}\left(L+\lambda_{j} J P-N, \Omega, 0\right)$. By Theorem 2.1 of [11], we have

$$
\operatorname{deg}\left(L+\lambda_{j} J P-N, \Omega, 0\right) \subseteq \operatorname{deg}\left(L+\lambda_{j} J P-N, \Omega_{1}, 0\right)+\operatorname{deg}\left(L+\lambda_{j} J P-N, \Omega_{2}, 0\right)
$$

for $j=1,2, \ldots$ Thus (1) follows from (2.2).

(2). Since $0 \notin \overline{\cup_{t \in[0,1]}(L-H(t, \cdot))(D(L) \cap \partial \Omega)}$, there exists $\lambda_{1}>0$ such that $0 \notin$ $\cup_{t \in[0,1]}(L+\lambda J P-H(t, \cdot))(\partial \Omega \cap D(L))$ for $\lambda \in\left(0, \lambda_{1}\right)$. By Theorem 2.1 of $[11], \operatorname{deg}(L+$ $\lambda J P-H(t, \cdot), \Omega, 0)$ does not depend on $t \in[0,1]$ for $\lambda \in\left(0, \min \left\{\lambda_{0}, \lambda_{1}\right\}\right)$. So $(2)$ follows from (2.2).

(3). If $\operatorname{deg}_{\Gamma_{0}}(L-N, \Omega, 0) \neq\{0\}$, then there exists $0 \neq m \in \operatorname{deg}_{\Gamma_{0}}(L-N, \Omega, 0)$, so there exists $\lambda_{j} \rightarrow 0^{+}$such that $m \in \operatorname{deg}\left(L+\lambda_{j} J P-N, \Omega, 0\right)$. Therefore $\left(L+\lambda_{j} J P-\right.$ $N) x$ has a solution in $\Omega \cap D(L), j=1,2, \ldots$ By letting $j \rightarrow \infty$, we obtain $0 \in$ $\overline{(L-N)(D(L) \cap \Omega)}$.

(4). We leave the proof to the reader.

(5). $L+\lambda J P$ is A-proper with respect to $\Gamma_{\lambda, L}$, and $0 \notin(L+\lambda J P)(\partial \Omega \cap D(L))$ for all $\lambda>0$. Since $L+\lambda J P$ is bijective, $\operatorname{deg}(L+\lambda J P, \Omega, 0) \subseteq\{ \pm 1\}$ for all $\lambda>0$. Thus we have

$$
\operatorname{deg}(L-N, \Omega, 0) \subseteq\{ \pm 1\}
$$

THEOREM 2.5. Let $X, Y$ be real separable Banach spaces, and $\left(Y_{n}, Q_{n}\right)$ a projectionally complete scheme for $Y$, and let $L: D(L) \subset X \rightarrow Y$ be a Fredholm mapping of zero index type, $0 \in \Omega \subset X$ a bounded subset, and $N: \bar{\Omega} \rightarrow Y$ a continuous compact mapping. Suppose the following conditions are satisfied

(1) $0 \notin \overline{(L-N)(\partial \Omega \cap D(L))}$;

(2) $0 \notin \overline{Q N(\partial \Omega \cap D(L))}$.

Then $\operatorname{deg}(L-N, \Omega, 0)=\operatorname{deg}(L-Q N, \Omega, 0)$.

Proof. For each $\lambda \in\left(0, \lambda_{0}\right)$, a similar proof to Proposition 1.11 shows that $\{L+$ $\lambda J P-t N-(1-t) Q N\}_{t \in[0,1]}$ is a homotopy of A-proper maps with respect to $\Gamma_{\lambda, L}$.

Now we claim that $0 \notin \overline{\cup_{t \in[0,1]}(L-t N-(1-t) Q N)(D(L) \cap \partial \Omega)}$.

If this is not true, then there exist $t_{j} \in[0,1]$ with $t_{j} \rightarrow t_{0}, x_{j} \in \partial \Omega \cap D(L)$, such that $L x_{j}-t_{j} N x_{j}-\left(1-t_{j}\right) Q N x_{j} \rightarrow 0$.

Case (1): if $t_{0}=1$, then $L x_{j}-N x_{j} \rightarrow 0$, which is a contradiction to assumption (1).

Case (2): if $t_{0} \neq 1$, then $Q L x_{j}-Q N x_{j} \rightarrow 0$, thus we have $Q N x_{j} \rightarrow 0$ and $x_{j} \in D(L)$, which is a contradiction to assumption (2). 
By (2) of Theorem 2.4, we obtain $\operatorname{deg}(L-N, \Omega, 0)=\operatorname{deg}(L-Q N, \Omega, 0)$.

Finally, let $L: D(L) \subseteq X \rightarrow Y$ be a Fredholm mapping of index zero type, $\Gamma_{0}=$ $\left(X_{n}, P_{n}\right)$ a projectionally complete scheme for $X$, and $\Omega \subset X$ an open bounded subset, and let $N: \bar{\Omega} \rightarrow Y$ be a mapping such that $I-(L+\lambda J P)^{-1}(N+\lambda J P)$ is an A-proper map with respect to $\Gamma_{0}$ for some $\lambda>0$. One can easily see that $0 \in L x-N x$ iff $0 \in$ $\left(I-(L+\lambda J P)^{-1}(N+\lambda J P)\right) x$. Assume that $0 \notin(L-N)(\partial \Omega \cap D(L))$. Then $0 \notin(I-$ $\left.(L+\lambda J P)^{-1}(N+\lambda J P)\right)(\partial \Omega)$ for all $\lambda>0$, and we define a generalized degree

$$
\operatorname{deg}_{\Gamma_{0}}(L-N, \Omega, 0)=\cup_{0<\lambda} \operatorname{deg}\left(I-(L+\lambda J P)^{-1}(N+\lambda J P), \Omega, 0\right),
$$

where $\operatorname{deg}\left(I-(L+\lambda J P)^{-1}(N+\lambda J P), \Omega, 0\right)$ is the generalized degree for A-proper maps if $I-(L+\lambda J P)^{-1}(N+\lambda J P)$ is A-proper with respect to $\Gamma_{0}$, otherwise $\operatorname{deg}(I-$ $\left.(L+\lambda J P)^{-1}(N+\lambda J P), \Omega, 0\right)=\emptyset$.

THEOREM 2.6. The generalized degree defined by (2.3) has the following properties.

(1) If $\Omega_{1}$ and $\Omega_{2}$ are disjoint open subsets of $\Omega$ such that $0 \notin(L-N)(D(L) \cap$ $\overline{\left.\Omega \backslash\left(\Omega_{1} \cup \Omega_{2}\right)\right)}$, then

$$
\operatorname{deg}_{\Gamma_{0}}(L-N, \Omega, 0) \subseteq \operatorname{deg}_{\Gamma_{0}}\left(L-N, \Omega_{1}\right)+\operatorname{deg}_{\Gamma_{0}}\left(L-N, \Omega_{2}, 0\right) .
$$

(2) If $H(t, x):[0,1] \times \bar{\Omega} \rightarrow Y$ satisfies $0 \notin \cup_{t \in[0,1]}(L-H(t, \cdot))(D(L) \cap \partial \Omega)$, and $\left\{I-(L+\lambda J P)^{-1}(H(t, \cdot)+\lambda J P)\right\}_{t \in[0,1]}$ is a homotopy of A-proper maps with respect to $\Gamma_{0}$ for all $\lambda>0$, then $\operatorname{deg}_{\Gamma_{0}}(L-H(t, \cdot), \Omega, 0)$ does not depend on $t \in[0,1]$.

(3) If $\operatorname{deg}_{\Gamma_{0}}(L-N, \Omega, 0) \neq\{0\}$, then $0 \in(L-N)(D(L) \cap \Omega)$.

(4) If $\Omega$ is a symmetric neighbourhood of 0 , and $N: \bar{\Omega} \rightarrow Y$ is an odd mapping such that $I-(L+\lambda J P)^{-1}(N+\lambda J P)$ is A-proper with respect to $\Gamma_{0}$ for some $\lambda>0$, and $0 \notin(L-N)(\partial \Omega \cap D(L))$, then $\operatorname{deg}_{\Gamma_{0}}(L-N, \Omega, 0)$ does not contain even numbers.

Proof. The proof is standard. We prove (2) and omit the others. Since $0 \notin$ $\cup_{t \in[0,1]}(L-H(t, \cdot))(D(L) \cap \partial \Omega)$, it follows that $0 \notin \cup_{t \in[0,1]}\left(I-(L+\lambda J P)^{-1}(H(t, \cdot)+\right.$ $\lambda J P))(\partial \Omega)$ for all $\lambda>0$. By Theorem 2.1 of [12], we know that

$$
\operatorname{deg}\left(I-(L+\lambda J P)^{-1}(H(t, \cdot)+\lambda J P), \Omega, 0\right)
$$

does not depend on $t \in[0,1]$ for each $\lambda>0$. Thus (2) follows from (2.3).

THEOREM 2.7. Suppose that $(L+\lambda J P)^{-1}: Y \rightarrow X$ is a continuous compact mapping for each $\lambda>0$, and $0 \in \Omega \subset X$ is an open bounded subset, $N: \bar{\Omega} \rightarrow Y$ is a continuous bounded mapping such that $L x \neq N x$, and $Q N x \neq \eta J P x$ for all $x \in \partial \Omega \cap D(L), \eta>0$, where $P, Q$ are projections as in section 1. Then $\operatorname{deg}(L-N, \Omega, 0)=\{1\}$.

Proof. Let $\Gamma_{0}=\left(X_{n}, P_{n}\right)$ be a projectionally complete scheme for $X$. Since $(L+$ $\lambda J P)^{-1}: Y \rightarrow X$ is continuous and compact for each $\lambda>0$, it follows that $\{I-(L+$ $\left.\lambda J P)^{-1} t(N+\lambda J P)\right\}_{t \in[0,1]}$ is a homotopy of A-proper maps with respect to $\Gamma_{0}$. We claim that $x \neq(L+\lambda J P)^{-1} t(N+\lambda J P) x$ for all $(t, x) \in[0,1] \times(\partial \Omega \cap D(L)), \lambda>0$. If this is not true, then there exist $\lambda_{0}>0,\left(t_{0}, x_{0}\right) \in[0,1) \times \partial \Omega$ such that $x_{0}=(L+$ $\left.\lambda_{0} J P\right)^{-1} t_{0}\left(N x_{0}+\lambda J P x_{0}\right)$. Thus we have $x_{0} \in D(L)$, and

$$
L x_{0}+\lambda_{0} J P x_{0}=t_{0}\left(N x_{0}+\lambda_{0} J P x_{0}\right) .
$$

Obviously, $t_{0} \neq 1$, therefore $\left(1-t_{0}\right) \lambda_{0} J P x_{0}=t_{0} Q N x_{0}$, which is a contradiction to one of our assumptions. Consequently, the A-proper degree $\operatorname{deg}\left(I-(L+\lambda J P)^{-1}(N+\right.$ 
$\lambda J P), \Omega, 0)=\operatorname{deg}(I, \Omega, 0)=\{1\}$. By (2.3), we obtain

$$
\operatorname{deg}_{\Gamma_{0}}(L-T, \Omega, 0)=\{1\} .
$$

COROLlary 2.8. Suppose that $H$ is a separable Hilbert space, and $(L+\lambda J P)^{-1}$ : $H \rightarrow X$ is a continuous compact mapping for each $\lambda>0$, and $0 \in \Omega \subset X$ is an open bounded subset, $N: \bar{\Omega} \rightarrow H$ is a continuous bounded mapping such that Lx $\neq N x$ for all $x \in \partial \Omega \cap D(L), Q N x \neq 0$ for $x \in \partial \Omega \cap D(L) \cap \operatorname{Ker}(P),(Q N x, J P x)<0$ for all $x \in \partial \Omega \cap D(L) \cap(\operatorname{Ker}(P))^{c}$, where $P, Q$ are projections as in section 1 . Then $\operatorname{deg}(L-N, \Omega, 0)=\{1\}$.

Proof. From our assumptions, we have $Q N x \neq \eta J P x$ for all $x \in \partial \Omega \cap D(L), \eta>0$. Thus the conclusion follows from Theorem 2.7.

3. An Example. Consider the following wave equation

$$
\left\{\begin{array}{l}
u_{t t}(t, x)-u_{x x}(t, x)-h(u(t, x))=f(t, x), \quad t \in(0,2 \pi), \quad x \in(0, \pi), \\
u(t, 0)=u(t, \pi)=0, \quad t \in(0,2 \pi), \\
u(0, x)=u(2 \pi, x), \quad x \in(0, \pi)
\end{array}\right.
$$

where $h: R \rightarrow R$ is a continuous function satisfying

$$
|h(u)| \leq \delta|u|+\gamma
$$

and $f(\cdot) \in L^{2}((0,2 \pi) \times(0, \pi))$, where $\delta>0, \gamma>0$ are constants.

We say $u \in L^{2}((0,2 \pi) \times(0, \pi))$ is a weak solution of $(\mathrm{E} 3.1)$ if

$$
\left(u, v_{t t}-v_{x x}\right)-(h(u(t, x)), v)=(f(t, x), v)
$$

for all $v \in C^{2}([0,2 \pi] \times[0, \pi])$ with $v(t, 0)=v(t, \pi)=0$ for $t \in[0,2 \pi]$, and $v(2 \pi, x)=$ $v(0, x)$ for $x \in[0, \pi]$.

Let $L: D(L) \subset L^{2}((0,2 \pi) \times(0, \pi)) \rightarrow L^{2}((0,2 \pi) \times(0, \pi))$ be the wave operator $L u=u_{t t}-u_{x x}$. Then it is well known that $L$ is self-adjoint, densely defined, closed, and $\operatorname{Ker}(L)$ is infinite dimensional with $\operatorname{Ker}(L)^{\perp}=\operatorname{Im}(L)$. Thus $L$ is a Fredholm mapping of zero index type. Let $P: L^{2}((0,2 \pi) \times(0, \pi)) \rightarrow \operatorname{Ker}(L)$ be the projection, then $(L+\lambda P)^{-1}: L^{2}((0,2 \pi) \times(0, \pi)) \rightarrow D(L)$ is compact for all $\lambda>0$.

Let $N: L^{2}((0,2 \pi) \times(0, \pi)) \rightarrow L^{2}((0,2 \pi) \times(0, \pi))$ be defined by $N u(t, x)=$ $h(u(t, x))+f(t, x)$ for $u(t, x) \in L^{2}((0,2 \pi) \times(0, \pi))$. By $(3.1), N$ is a bounded continuous mapping. For each $\eta>0$, consider the following equation

$$
\left\{\begin{array}{l}
u_{t t}(t, x)-u_{x x}(t, x)+\eta u(t, x)-h(u(t, x))=f(t, x), \quad t \in(0,2 \pi), \quad x \in(0, \pi), \\
u(t, 0)=u(t, \pi)=0, \quad t \in(0,2 \pi) \\
u(0, x)=u(2 \pi, x), \quad x \in(0, \pi)
\end{array}\right.
$$

where $h, f$ are as in (E 3.1). Let $u_{\eta}$ be the weak solution of (E 3.2) if it exists, and we set $S=\left\{u_{\eta}: \eta>0\right\}$. Now we have the following alternative result.

THEOREM 3.1. S is unbounded in $L^{2}((0,2 \pi) \times(0, \pi))$ or $(\mathrm{E} 3.1)$ has a weak solution. 
Proof. We may assume that $S$ is bounded in $L^{2}((0,2 \pi) \times(0, \pi))$. So there exists $r_{0}>0$ such that

$$
\left\|u_{\eta}\right\|_{L^{2}}<r_{0}, \text { for all } u_{\eta} \in S \text {. }
$$

Let $\Omega=\left\{u(t, x) \in L^{2}((0,2 \pi) \times(0, \pi)):\|u\|_{L^{2}}<r_{0}\right\}$. By (3.2), we know $P N u \neq \eta P u$ for all $u \in C^{2}([0,2 \pi] \times[0, \pi]) \cap \partial \Omega$, and $\eta>0$. We may assume that $L u \neq N u$ for all $u \in C^{2}([0,2 \pi] \times[0, \pi]) \cap \partial \Omega$.

By Theorem 2.7, we have $\operatorname{deg}(L-N, \Omega, 0)=\{1\}$, thus (E 3.1) has a weak solution.

\section{REFERENCES}

1. J. Berkovits and V. Mustonen, An extension of Leray-Schauder degree and applications to nonlinear wave equations, Diff. Int. Equat. 3 (1990), 945-963.

2. H. Brézis, Periodic solutions of nonlinear vibrating strings and duality principles, Bull. Amer. Math. Soc. 8 (1983), 409-426.

3. F. E. Browder and W. V. Petryshyn, Approximation methods and the generalized topological degree for nonlinear mappings in Banach spaces, J. Funct. Anal. 3 (1969), 217 274.

4. M. Feckan and R. Kollar, Discontinuous wave equations and a topological degree for some classes of multi-valued mappings, Appl. Math. 44 (1999), 15-32.

5. P. M. Fitzpatrick, A generalized degree for uniform limits of A-proper mappings, $J$. Math. Anal. Appl. 35 (1971), 536-552.

6. R. E. Gaines and J. Mawhin, Coincidence degree and nonlinear differential equations, Lecture Notes in Mathematics No. 568 (Springer-Verlag, 1977).

7. J. Mawhin, Nonlinear perturbations of Fredholm mappings in normed spaces and applications to differential equations, Univ. de Brasilia, Trabalho de Matematica, No. 61 (1974).

8. J. Mawhin Topological degree methods in nonlinear boundary value problems (Amer. Math. Soc. Providence, RI, 1979).

9. W. V. Petryshyn, Fredholm theory for abstract and differential equations with noncompact nonlinear perturbations of Fredholm maps, J. Math. Anal. Appl. 72 (1979), 472499.

10. W. V. Petryshyn, Using degree theory for densely defined A-proper maps in the solvability of semilinear equations with unbounded and noninvertible linear part, Nonlinear Anal. 4 (1980), $259-281$.

11. W. V. Petryshyn and Z. S. Yu, Existence theorems for higher-order nonlinear periodic boundary value problems, Nonlinear Anal. 6 (1982), 943-969.

12. W. V. Petryshyn, Generalized topological degree and semilinear equations (Cambridge University Press, 1995). 